\title{
Exploring the pattern of Lepton Flavour Universality viola- tion at hadron colliders
}

\author{
Abhishek M. Iyer ${ }^{1, *}$ \\ ${ }^{1}$ INFN-Sezione di Napoli, Via Cintia, 80126 Napoli, Italia
}

\begin{abstract}
We discuss the $B$ anomalies in scenario with warped extra dimensions characterized by additional neutral heavy bosons. Depending on the coupling of the fermions (leptons in particular) different scenarios for the explanation of the anomalies can be obtained. We focus on a specific case which involves contributions due to both the electron and the muon leading to a four dimensional fits in the space of Wilson coefficients. With this as a motivation, in a generic $Z^{\prime}$ we develop correlations with direct search experiments, wherein the pattern of the Wilson coefficients can be uniquely extracted at the LHC.
\end{abstract}

\section{Introduction}

Flavour observables offer a strong probe towards the indirect detection for NP effects with flavour structures different from that of the SM. The recent measurements of the semi-leptonic decay of the $B$ mesons in the $b \rightarrow$ sll sector has generated a lot of interest. The observed anomalies were quoted through the measurement of the following hadronicall clean ratio [1]

$$
\begin{aligned}
R_{K} & =\left.\frac{\mathcal{B}\left(B^{+} \rightarrow K^{+} \mu^{+} \mu^{-}\right)}{\mathcal{B}\left(B^{+} \rightarrow K^{+} e^{+} e^{-}\right)}\right|_{q^{2}=1-6 \mathrm{GeV}^{2}} \\
& =0.745_{-0.074}^{+0.090}(\text { stat }) \pm 0.036 \text { (syst) }
\end{aligned}
$$

The SM expectation for the ratio is $R_{K}^{S M}=1.003$ [2] indicating a a $\sim 2.6 \sigma$ deviation as a possible evidence of lepton flavour non-universality. Another measurement through the ratio $R\left(K^{*}\right)$ exhibited a pattern similar to $R(K)$ as

$$
\begin{aligned}
R_{K^{*}} & =\frac{\mathcal{B}\left(B^{0} \rightarrow K^{* 0} \mu^{+} \mu^{-}\right)}{\mathcal{B}\left(B^{0} \rightarrow K^{* 0} e^{+} e^{-}\right)} \\
& =0.660_{-0.070}^{+0.110}(\text { stat }) \pm 0.024(\text { syst }), \quad \text { low } q^{2} \\
& =0.685_{-0.069}^{+0.113}(\text { stat }) \pm 0.047(\text { syst }), \quad \text { mid } q^{2}
\end{aligned}
$$

For this, the low $q^{2}$ corresponds to $0.045 \leq q^{2} \leq 1.1 \mathrm{GeV}^{2}$ while mid $q^{2}$ corresponds to $1.1 \leq q^{2} \leq 6.0 \quad \mathrm{GeV}^{2}$. The corresponding SM values for the $q^{2}$ bins are: $R_{K^{*}}^{S M} \simeq 0.93$ for low $q^{2}$ while $R_{K^{*}}^{S M}=1$ elsewhere. This translates into a $2.4 \sigma$ and $\sim 2.5 \sigma$ deviation for the

*e-mail: iyera@na.infn.it 
low $q^{2}$ and for medium $q^{2}$ bins respectively. These deviations are typically parametrized by additional contributions to the Wilson coefficients $C_{i}$ of the following effective operators: [3]:

$$
\mathcal{L} \supset \frac{G_{F} \alpha}{\sqrt{2} \pi} \sum_{i} C_{i} O_{i}
$$

where $C_{i}=C_{i}^{S M}+C_{i}^{N P}$.

$$
\begin{array}{rll}
O_{9} & =\left(\bar{s}_{L} \gamma^{\mu} b_{L}\right)\left(\bar{l} \gamma_{\mu} l\right) & O_{9^{\prime}}=\left(\bar{s}_{R} \gamma^{\mu} b_{R}\right)\left(\bar{l} \gamma_{\mu} l\right) \\
O_{10}=\left(\bar{s}_{L} \gamma^{\mu} b_{L}\right)\left(\bar{l} \gamma_{\mu} \gamma^{5} l\right) & O_{10^{\prime}}=\left(\bar{s}_{R} \gamma^{\mu} b_{R}\right)\left(\bar{l} \gamma_{\mu} \gamma^{5} l\right)
\end{array}
$$

Here $C_{i}^{N P}$ determines the NP contributions to the Wilson coefficients and $l$ denotes a lepton. In this talk we will consider a scenario where NP effects exits in both the muon and the electron sector due to the exchange of the neutral heavy boson. These exotic states can arise to gauge extensions of the SM. However, we consider these fields arising as Kaluza-Klein (KK) excitations of extra-dimensional models. In particular we consider a model with a single warped extra-dimension with the following line element: [5]:

$$
d s^{2}=e^{-2 A(y)} \eta_{\mu \nu} d x^{\mu} d x^{v}-d y^{2}
$$

where $A(y)=k|y|, k \sim \frac{M_{P l}}{4 \pi}$ and $0 \leq y \leq \pi R$. The coordinates $y=0, \pi R$ correspond to the location of the $(U V, I R)$ brane respectively. The minimal model, with brane localized fields and SM gauge symmetry is severely constrainted by EWPT. A generalization of this setup with bulk fields and a gauged custodial symmetry [6] was then proposed which not only relaxed the bounds from large contributions to the $T$ parameter but embedding the fermions in non singlet representations of the gauge group served to evade constraints from $Z \rightarrow f \bar{f}$. As mentioned earlier, the presence of bulk fields leads to KK excitations in the effective low energy theory. Along with the KK excitations of the SM $W, Z$, the model is also characterized by additional 'custodial' heavy gauge bosons thereby leading to a distinct phenomenology, in the flavour sector in particular. A detailed analysis of different flavour transitions in this setup was considered in [7-10]. We explore the parameter space admitted by the current anomalies [11]. We demonstrate fits with the following two scenarios which are characterized by different extent of contributions to the Wilson coefficients of the electron and the muon. We then setup a high $p_{T}$ collider probe for any generic $Z^{\prime}$ scenario wherein it is possible to extract the pattern of the Wilson coefficients.

The note is organized as follows: In Section 2 we compute the fits for the anomalies in $b \rightarrow$ sll processes for the two different scenarios. In Section 3 we develop a strategy to extract the Wilson coefficients at collider and we finally conclude.

\section{$2 b \rightarrow$ sll processes: $B$ anomalies}

In bulk custodial models in RS, neutral currents at tree level receive contribution to the four

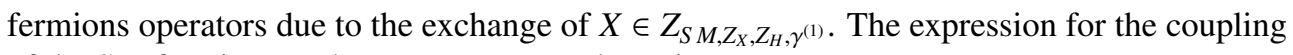
of the SM fermions to these NP states can be written as:

$$
\mathcal{L}_{N P} \subset X_{\mu}\left[\alpha_{L}^{b s}(X)\left(\bar{s}_{L} \gamma^{\mu} b_{L}\right)+\alpha_{R}^{b s}(X)\left(\bar{s}_{R} \gamma^{\mu} b_{R}\right)+\bar{\mu}\left(\alpha_{V}^{l}(X) \gamma^{\mu}-\alpha_{A}^{l}(X) \gamma^{\mu} \gamma^{5}\right) \mu\right]
$$

where $\alpha_{V, A}^{l}(X)=\frac{\alpha_{L}^{l}(X) \pm \alpha_{R}^{l}(X)}{2}$. $\alpha$ gives the coupling in the zero mode fermions to the gauge bosons in the mass basis of the former. The Wilson co-efficients for each gauge field $X$ can then be written as:

$$
\Delta C_{9}=-\frac{\sqrt{2} \pi}{M_{X}^{2} G_{F} \alpha} \alpha_{L}^{b s}(X) \alpha_{V}^{l}(X), \quad \Delta C_{10}=\frac{\sqrt{2} \pi}{M_{X}^{2} G_{F} \alpha} \alpha_{L}^{b s}(X) \alpha_{A}^{l}(X),
$$



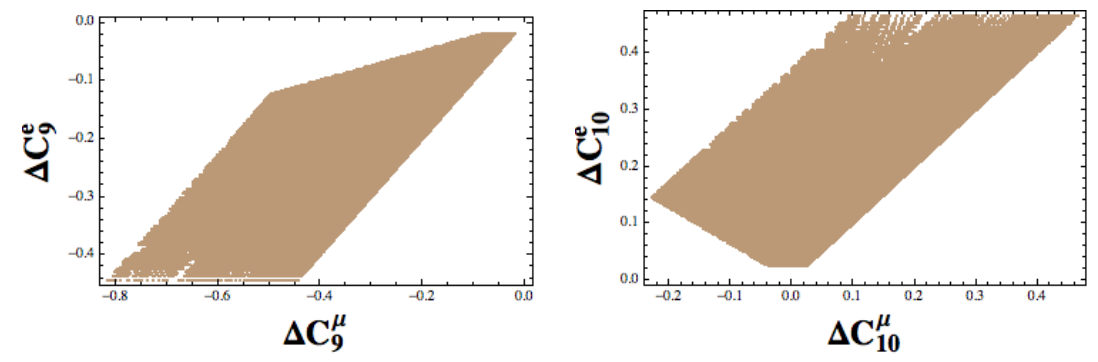

Figure 1. Scenario A: Plot show the correlation in the $C_{9}$ and $C_{10}$ parameter plane for both the electron and the muon. The bottom row gives the range of $c_{b_{L}}$ if $-1.4<C_{10}^{\mu}<-0.7$. We use $M_{K K}=3 \mathrm{TeV}$

The primed operators vanish due the presence of an accidental $U(3)$ in the coupling of the down quark singlets to the gauge KK states. Assuming the the up-sector quark to be in the mass-diagonal basis, down quark rotation matrices are simply $D_{L} \sim V_{C K M}$. We now discuss the following two scenarios:

1) Scenario A: In this scenario the lepton singlets have relatively larger coupling to the NP than the doublets. The doublets have universal bulk wave function with $c>0.5$. The scanning range for the doublets and the muon singlets is chosen to be $0.45<c<0.55$. Thus even though this scenario can admit a relatively larger coupling of muon singlets to NP than the corresponding doublets, the other possibility still exists. The ranges chosen for $c$ parameter scan is: $c_{Q_{3}} \in[0.4,0.5], c_{\mu_{L}}=c_{L} \in[0.45,0.55]$ and $c_{\mu_{R}} \in[0.45,0.55]$. The scan in this scenario has a couple of unique features: The $\Delta C_{9}^{\mu}-\Delta C_{10}^{\mu}$ plot in Fig. 1 has a feature where $\Delta C_{10}^{\mu}$ may vanish while the contribution to $\Delta C_{9}^{\mu}$ is considerable. This is an artifact of the similar scanning range for the lepton doublets and the muon singlets. This corresponds to the explicit case where $\alpha_{L}^{l}(X)=\alpha_{R}^{l}(X)$. Corresponding to this, we discuss two minor possibilities:

a) $\Delta C_{10}^{\mu}$ vanishes and the contributions to $\Delta C_{9,10}^{e}$ are very small, thereby reducing the analysis to a one-dimensional fit with contributions mainly from $\Delta C_{9}^{\mu}$. The best fit for this case is $\Delta C_{9}^{\mu}=-1.5$ with a $2 \sigma$ region $[-2.9,-1.73][4,17]$. From the bottom plot of Fig. 1 , we find that we can obtain a fit only in the $3 \sigma$ region where the electron contribution is negligible.

b) The tight condition 1-D scenario can be further relaxed, once the electrons also contribute considerably. This opens up the possibility of a four dimensional fit in the plane of Wilson coefficients and the results for this case are shown in Fig.1. Lower row of Fig. 1 gives the different correlations: $C_{9}^{\mu}-C_{9}^{e}$ (top) and $C_{10}^{\mu}-C_{10}^{e}$ (bottom). The 2- $\sigma$ regions for a $4 \mathrm{D}$ fit to the data is [12]

$$
\frac{C_{9}^{\mu}}{C_{9}^{S M}} \in[-0.33,0.06] ; \quad \frac{C_{9}^{e}}{C_{9}^{S M}} \in[-2.23,0.74] ; \quad \frac{C_{10}^{\mu}}{C_{10}^{S M}} \in[-0.29,0.14] ; \quad \frac{C_{10}^{e}}{C_{10}^{S M}} \in[-2.60,0.60] .
$$

With a four dimensional fit, it is relatively easier to find solutions which satisfy the above regions.

The non-negligible values of the $\Delta C^{e}$ is due to left doublets having $c \sim 0.5$ thereby resulting in a mildly larger coupling to the NP states than would be expected of states having $c \geq 0.55$. For these choices of $c$ parameters corresponding to the values in Fig.1, fitting the muon mass requires choosing the $O(1)$ Yukawa $\sim 0.03$. Though slightly fine tuned 

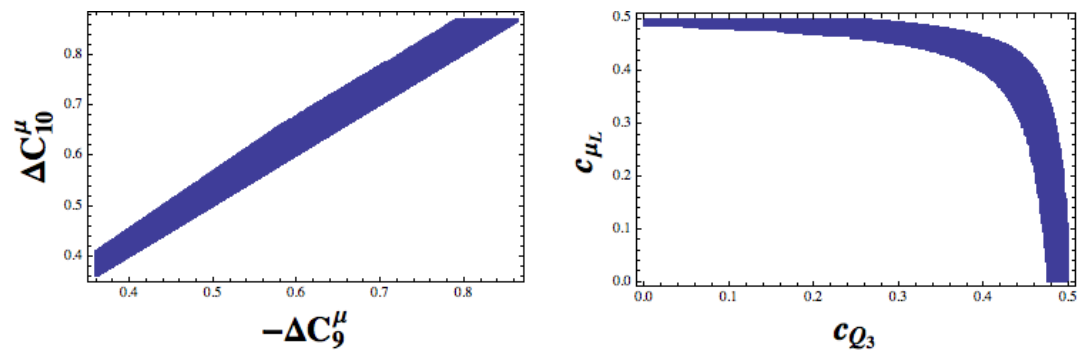

Figure 2. Scenario $\mathbf{B}$ : Left plot gives the distribution for $\Delta C_{9}$ and $\Delta C_{10}$. The corresponding $c$ parameters ranges are given in the right plot.

with regards to the fit to the muon mass, this scenario is more favorable with regards to an anarchic neutrino mixing matrix but also in suppressing FCNC in the lepton sector through the implementation of 5D MFV.

2) Scenario B: The non-universality in this case exists in the coupling of the lepton doublets to NP. Further, we assume that the $\tau$ doublets to be relatively closer to the IR brane than the $\mu$ doublets $\left(c_{\tau_{L}}<c_{\mu_{L}}\right)$. All the lepton singlets and the electron doublet satisfy $c>0.55$. This results in $\Delta C_{9,10}^{e}$ much smaller than $\Delta C_{9,10}^{\mu}$, with its magnitude being at most $\sim 0.2$. For most of the region, it effectively reduces this to a 2-D fit where the value of $C_{9,10}^{e}$ is an order of magnitude less,

Top plot of Fig.2 gives the correlation in the $\Delta C_{9}^{\mu}-\Delta C_{10}^{\mu}$ plane which correspond to points which satisfy $0.36<\left|\Delta C_{9,10}^{\mu}\right|<0.87$. The correlation also shows that there exists solutions for which $\Delta C_{9}^{\mu}=-\Delta C_{10}^{\mu}$ reducing it to a 1-D fit as discussed in [16, 17]. The two relevant parameters for the fits to the $B$-anomalies in this case are $c_{Q_{3}}-c_{\mu_{L}}$ and the correlation is shown in the bottom plot of Fig. 2.

\section{Correlating the pattern of solutions to high $p_{T}$ searches at colliders}

In the previous section we demonstrated two different scenarios in the same model which can explain the $B$ anomalies. Scenario A in particular included contributions due to both the electron and the muon. They were associated with braod spread in the ranges for the Wilson coefficients. We now develop a correlation wherein the pattern involving electrons and/or muons can be probed at high energy $p$ - $p$ collisions. To begin with, we consider the following neutral current Lagrangian:

$$
\mathcal{L}_{e f f}=\frac{\lambda_{b s}}{M^{2}}\left(\bar{s} \gamma_{\mu} b\right)\left[\lambda_{e}\left(\bar{e} \gamma^{\mu} e\right)+\lambda_{\mu}\left(\bar{\mu} \gamma^{\mu} \mu\right)+\lambda_{\tau}\left(\bar{\tau} \gamma^{\mu} \tau\right)\right]+\ldots
$$

Given the Lagrangian in Eq. 9, the Wilson-coefficients for the $R\left(K^{*}\right)$ anomalies are identified as:

$$
C_{9}^{e}=-\frac{\sqrt{2} \pi}{G_{F} \alpha} \frac{\lambda_{b s} \lambda_{e}^{V}}{M^{2}} \quad C_{9}^{\mu}=-\frac{\sqrt{2} \pi}{G_{F} \alpha} \frac{\lambda_{b s} \lambda_{\mu}^{V}}{M^{2}} \quad C_{10}^{e}=-\frac{\sqrt{2} \pi}{G_{F} \alpha} \frac{\lambda_{b s} \lambda_{e}^{A V}}{M^{2}} \quad C_{10}^{\mu}=-\frac{\sqrt{2} \pi}{G_{F} \alpha} \frac{\lambda_{b s} \lambda_{\mu}^{A V}}{M^{2}}
$$

where $\lambda^{V}=\frac{L+R}{2}$ and $\lambda^{A V}=\frac{L-R}{2}$ are the vector and axial vector coupling respectively of the leptons to $Z^{\prime} . L(R)$ is the coupling strength of doublets (singlets) to $Z^{\prime}$. In the first instance, 

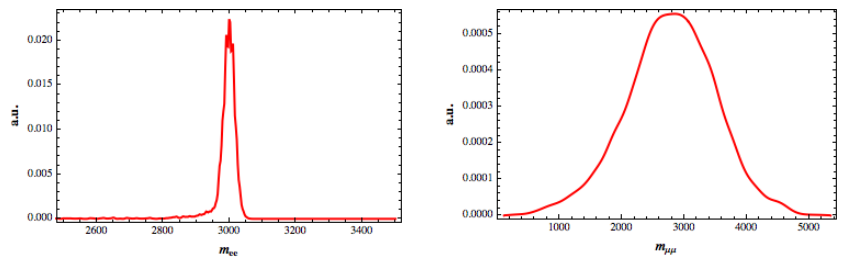

Figure 3. Mass reconstruction for the electron (left) and muon (right) for $M_{Z^{\prime}}=3 \mathrm{TeV}$

we assume $L=R$, implying the axial-vector couplings of the leptons to $Z^{\prime}$ vanish. In this case the ratio of $C_{9}$ for muons and electrons is simply $\frac{C_{\mu}^{9}}{C_{e}^{9}}=\frac{\lambda_{\mu}^{V}}{\lambda_{e}^{V}}$ where the quark dependence cancels out.

In a direct production of $Z^{\prime}$ in $p p$ collisions decaying into electrons and muons. The ratio of number di-muons to di-electrons at luminosity $\mathcal{L}$ is simply given as:

$$
\delta=\frac{\sigma_{Z^{\prime}}\left(\lambda_{\mu}^{V}\right)^{2} \mathcal{L} \epsilon_{\mu}}{\sigma_{Z^{\prime}}\left(\lambda_{e}^{V}\right)^{2} \mathcal{L} \epsilon_{e}}=\frac{N_{\mu}}{N_{e}} .
$$

In the event, the acceptance efficiency of the electrons and the muons are similar, then the ratio in Eq. 11 would exactly mirror the ratio of the Wilson coefficients. However, as shown in Table. 1, electrons and muons are characterized by different acceptance efficiencies. In evaluating the numbers in Table 1, the first row gives the number of events with atleast a single leptons and bottom row computes the number of leptons over a given threshold.

\begin{tabular}{|c|c|c|}
\hline & $\epsilon_{\mu}$ & $\epsilon_{e}$ \\
\hline Simple Isolation(>1 leptons) & 59.33 & 39.79 \\
Mass cuts $(>1000 \mathrm{GeV})$ & 58.79 & 39.61 \\
\hline
\end{tabular}

Table 1. Comparison of acceptance efficiencies for electrons and muons for $m_{Z^{\prime}}=3000 \mathrm{GeV}$

Note that the methodology of counting the number of di-leptons over a given threshold is rather unconventional. Traditional methods involve counting events in a given bin around a possible resonant mass. The electron and the muon are characterized by different reconstruction efficiencies. The latter is associated with the large smearing of the four moments especially at high $p_{T}$ while the electrons have a sharp reconstruction independent of the mass of the resonance. This is illustrated in Fig. 3. Correspondingly Fig. 4 gives the corresponding sensitivity for two different mass bins around the pole mass: $M_{Z^{\prime}} \pm 200 \mathrm{GeV}$ (left) and $M_{Z^{\prime}} \pm 500 \mathrm{GeV}$ (right). Note that the efficiency changes depending on the choice of bin due to different reconstruction efficiencies. As a result in order to overcome the difference in the reconstruction efficiency, we propose to simply count the di-lepton events over a given threshold.

Further to ensure best implementation of the ration in Eq. 11 at colliders, we must ensure it is due to difference in couplings of the $Z^{\prime}$ to the leptons and not due to different acceptance efficiency. Increasing the acceptance of the electrons can be achieved using the electron jet techniques and using the following substructure variables to identify the electron [22]:

A) Hadronic energy fraction: As electron deposit their energy in the $E-c a l$, the corresponding jets are associated with very small $H$-cal component of the energy. This helps in limiting 

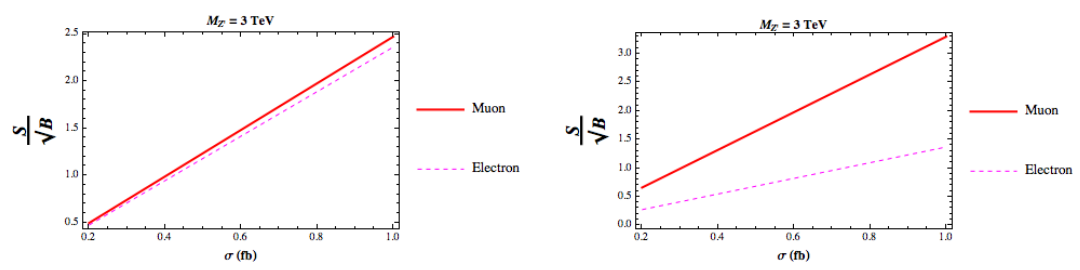

Figure 4. The plot gives the comparison of corresponding sensitivity as a function of $\sigma_{Z^{\prime}}$ at $\mathcal{L}_{\text {int }}=3000$ $\mathrm{fb}^{-1}$ for two different bin choices: The left column corresonds to a choice $M_{Z^{\prime}} \pm 200 \mathrm{GeV}$ and the right column corresponds to $M_{Z^{\prime}} \pm 500 \mathrm{GeV}$. Note that for all cases we assume $\operatorname{B} \cdot \operatorname{R}\left(Z^{\prime} \rightarrow \mu \mu\right)=4$ B.R $\left(Z^{\prime} \rightarrow e e\right)$

\begin{tabular}{|c|c|c|c|}
\hline$m_{Z^{\prime}}(\mathrm{GeV})$ & $\epsilon_{\mu}$ & $\epsilon_{e}$ (Electron jets) & $\epsilon_{\tau}$ (tau jets) \\
\hline 2000 & 71.45 & 64.75 & 31.25 \\
2500 & 66.35 & 63.06 & 37.28 \\
3000 & 58.79 & 60.37 & 40.88 \\
3500 & 51.68 & 59.50 & 43.98 \\
\hline
\end{tabular}

Table 2. Comparison of efficiencies of electron jets and muons for different $m_{Z^{\prime}}$ masses. A selectrion criteria of the $m_{\mu \mu}$ or $m_{j_{0} j_{1}}(>1000) \mathrm{GeV}$ is imposed. For the electron jets the QCD fake rate is $<1$ in $3 \times 10^{5}$ events. For $\tau$ jets the QCD fake rate is $0.2 \%$

QCD to a great extent and B) Tracks: We demand that the leading jet is associated with exactly one track, while the subleading jet may have 0 or 1 track. The latter condition facilitates in accepting events which would otherwise have been rejected by the standard electron identification criteria.

Table 2 gives a comparison of the muon and 'electron' efficiencies using electron jets. We find that the acceptance efficiencies are within $5-10 \%$ of each other. Thus any discrepancy in the observation of the ratio can be attributed to the difference in the couplings of the NP to electrons and muons. The third column of Table 2 gives the acceptance efficiency for tau jets. This technique can be used to extract information on $C_{9}^{\tau}$, though with a lesser accuracy than the leptons and have implications for processes like $B \rightarrow K^{*} \tau \tau$

\section{Conclusions}

We discussed a warped framework to explain the observations of lepton flavour universality violation in the semileptonic decay of $B$ mesons. One particular solution led to a case where both electron and muon contribute. This provided a motivation to extract the nature of Wilson coefficients at collider by probing the resonant decay of $Z$ ' into leptons. We propose a strategy to ensure that any observed deviations in the $N_{e e} / N_{\mu \mu}$ is primarily due to differential coupling of NP to leptons.

\section{Acknowledgements}

I would like to thank the organizers of QCD@work 2018 for the opportunity to present my work in the workshop. 


\section{References}

[1] R. Aaij et al. [LHCb Collaboration], Phys. Rev. Lett. 113, 151601 (2014) doi:10.1103/PhysRevLett.113.151601 [arXiv:1406.6482 [hep-ex]].

[2] M. Bordone, G. Isidori and A. Pattori, Eur. Phys. J. C 76, no. 8, 440 (2016) doi:10.1140/epjc/s10052-016-4274-7 [arXiv:1605.07633 [hep-ph]].

[3] G. Buchalla, A. J. Buras and M. E. Lautenbacher, Rev. Mod. Phys. 68, 1125 (1996) doi:10.1103/RevModPhys.68.1125 [hep-ph/9512380].

[4] L. S. Geng, B. Grinstein, S. Jäger, J. Martin Camalich, X. L. Ren and R. X. Shi, Phys. Rev. D 96, no. 9, 093006 (2017) doi:10.1103/PhysRevD.96.093006 [arXiv:1704.05446 [hep-ph]].

[5] L. Randall and R. Sundrum, Phys. Rev. Lett. 83, 3370 (1999) doi:10.1103/PhysRevLett.83.3370 [hep-ph/9905221].

[6] K. Agashe, A. Delgado, M. J. May and R. Sundrum, JHEP 0308, 050 (2003) doi:10.1088/1126-6708/2003/08/050 [hep-ph/0308036].

[7] M. Blanke, A. J. Buras, B. Duling, S. Gori and A. Weiler, JHEP 0903, 001 (2009) doi:10.1088/1126-6708/2009/03/001 [arXiv:0809.1073 [hep-ph]].

[8] M. Blanke, A. J. Buras, B. Duling, K. Gemmler and S. Gori, JHEP 0903, 108 (2009) doi:10.1088/1126-6708/2009/03/108 [arXiv:0812.3803 [hep-ph]].

[9] M. Bauer, S. Casagrande, U. Haisch and M. Neubert, JHEP 1009, 017 (2010) doi:10.1007/JHEP09(2010)017 [arXiv:0912.1625 [hep-ph]].

[10] S. Casagrande, F. Goertz, U. Haisch, M. Neubert and T. Pfoh, JHEP 1009, 014 (2010) doi:10.1007/JHEP09(2010)014 [arXiv:1005.4315 [hep-ph]].

[11] G. D'Ambrosio and A. M. Iyer, arXiv:1712.08122 [hep-ph].

[12] T. Hurth, F. Mahmoudi and S. Neshatpour, Nucl. Phys. B 909, 737 (2016) doi:10.1016/j.nuclphysb.2016.05.022 [arXiv:1603.00865 [hep-ph]].

[13] A. J. Buras, D. Buttazzo, J. Girrbach-Noe and R. Knegjens, JHEP 1511, 033 (2015) doi:10.1007/JHEP11(2015)033 [arXiv:1503.02693 [hep-ph]].

[14] T. K. Komatsubara, Prog. Part. Nucl. Phys. 67, 995 (2012) doi:10.1016/j.ppnp.2012.04.001 [arXiv:1203.6437 [hep-ex]].

[15] K. Shiomi [KOTO Collaboration], arXiv:1411.4250 [hep-ex].

[16] B. Capdevila, A. Crivellin, S. Descotes-Genon, J. Matias and J. Virto, arXiv:1704.05340 [hep-ph].

[17] W. Altmannshofer, P. Stangl and D. M. Straub, Phys. Rev. D 96, no. 5, 055008 (2017) doi:10.1103/PhysRevD.96.055008 [arXiv:1704.05435 [hep-ph]].

[18] G. Buchalla and A. J. Buras, Nucl. Phys. B 548, 309 (1999) doi:10.1016/S05503213(99)00149-2 [hep-ph/9901288].

[19] C. Patrignani et al. [Particle Data Group], Chin. Phys. C 40, no. 10, 100001 (2016). doi:10.1088/1674-1137/40/10/100001

[20] F. Mescia and C. Smith, Phys. Rev. D 76, 034017 (2007) doi:10.1103/PhysRevD.76.034017 [arXiv:0705.2025 [hep-ph]].

[21] G. Buchalla and A. J. Buras, Nucl. Phys. B 412, 106 (1994) doi:10.1016/05503213(94)90496-0 [hep-ph/9308272].

[22] F. Conventi, G. D 'Ambrosio, A. M. Iyer and E. Rossi et al., To appear [arXiv:1810.xxxx ].

[23] A. Chakraborty, A. M. Iyer and T. S. Roy, Nucl. Phys. B 932, 439 (2018) doi:10.1016/j.nuclphysb.2018.05.019 [arXiv:1707.07084 [hep-ph]]. 